\title{
A label-free surface-enhanced Raman spectroscopy biosensor for on- site breast cancer detection using human tears
}

Soogeun Kimª,1, Tae Gi Kim ${ }^{\mathrm{b}, 1}$, Soo Hyun Leec,1, Wansun Kimª, Ayoung Banga ${ }^{\mathrm{a}}$, Sang Woong Moon $^{b}$, Jeongyoon Song ${ }^{d}$, Jae-Ho Shin ${ }^{\text {* }}$, Jae Su Yuc*, Samjin Choia*

a. Department of Biomedical Engineering, College of Medicine, Kyung Hee University, Seoul 02447, South Korea

b. Department of Ophthalmology, College of Medicine, Kyung Hee University, Seoul 02447, South Korea

c. Department of Electronic Engineering, Institute for Wearable Convergence Electronics, Kyung Hee University, Gyeonggi-do 17104, South Korea

d. Department of Surgery, College of Medicine, Kyung Hee University, Seoul 02447, South Korea

\section{*Address for correspondence:}

Jae-Ho Shin, M.D. \& Ph.D., Jae Su Yu, Ph.D., Samjin Choi, Ph.D.

Department of Biomedical Engineering,

College of Medicine, Kyung Hee University

26, Kyungheedae-ro, Dongdaemun-gu,

Seoul 02447,

South Korea

Tel: +82 2961 0290;

fax: +82 29615515

E-mail addresses: pbloadsky@naver.com (J.H. Shin), jsyu@khu.ac.kr (J.S. Yu), medchoi@khu.ac.kr (S. Choi) 


\section{FE-SEM images of Au/HCP-PS monolayer on Si substrate}
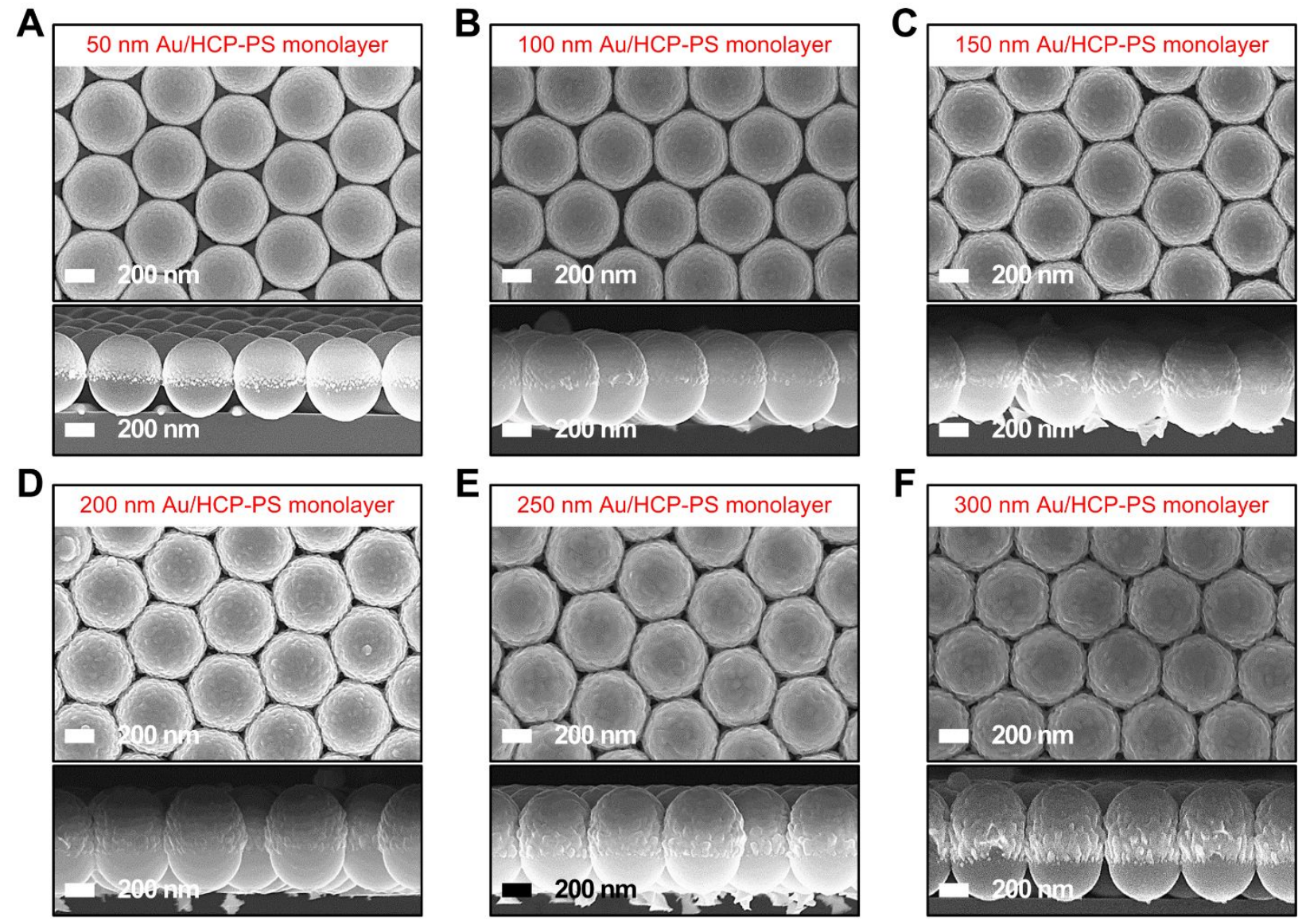

Figure S1. FE-SEM images of the Au/HCP-PS microsphere arrays on the Si substrate with Au thicknesses of (A) $50 \mathrm{~nm}$, (B) $100 \mathrm{~nm}$, (C) $150 \mathrm{~nm}$, (D) $200 \mathrm{~nm}$, (E) $250 \mathrm{~nm}$, and (F) $300 \mathrm{~nm}$. 


\section{AFM topographical images of Au/HCP-PS monolayer on Si substrate}

A
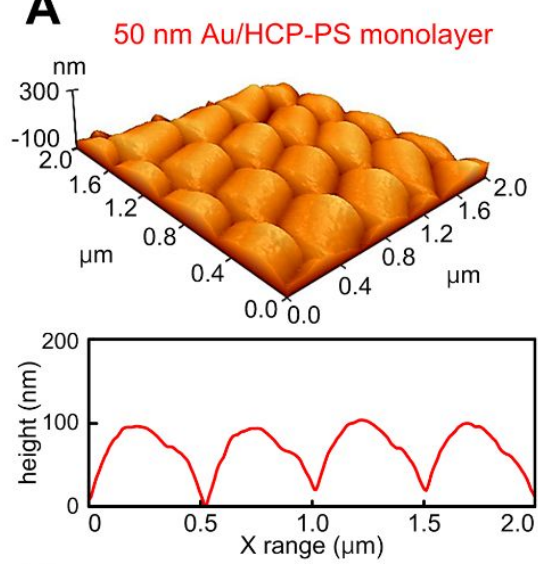

D $200 \mathrm{~nm}$ Au/HCP-PS monolayer
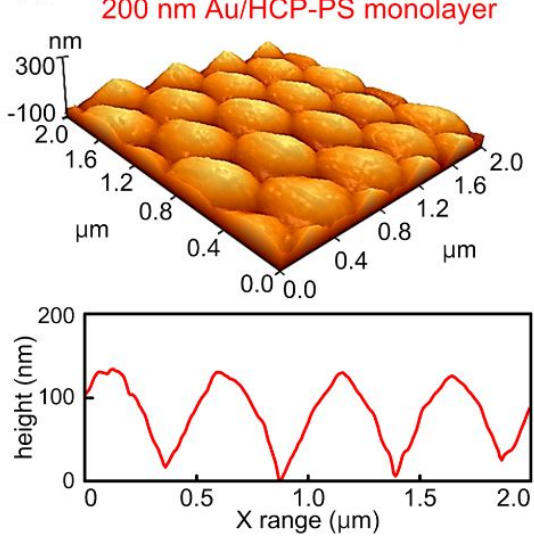

B
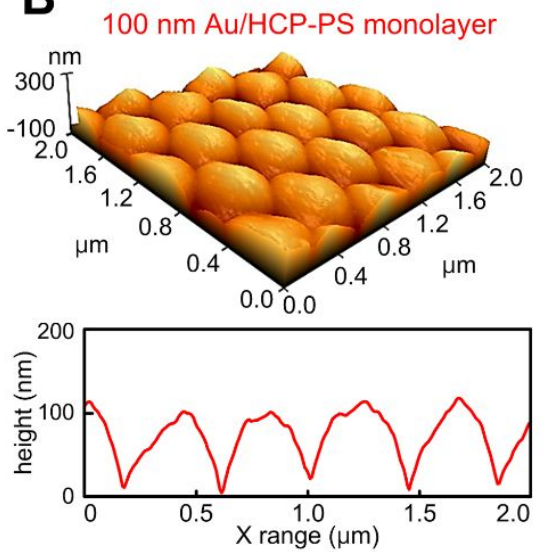

E
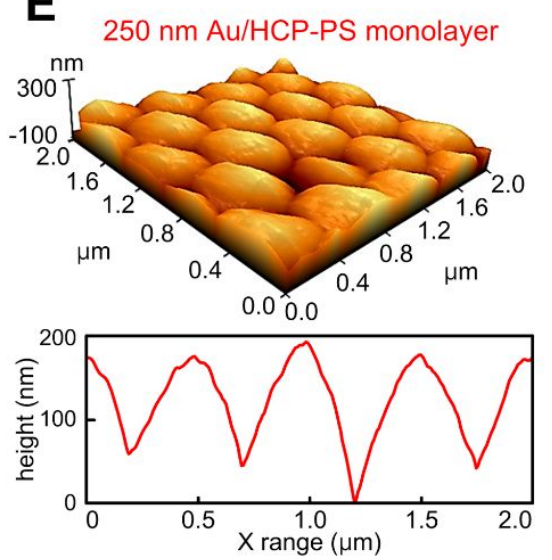

C
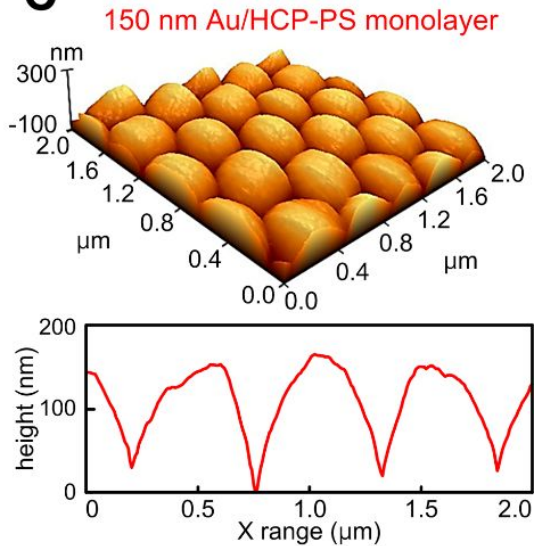

F
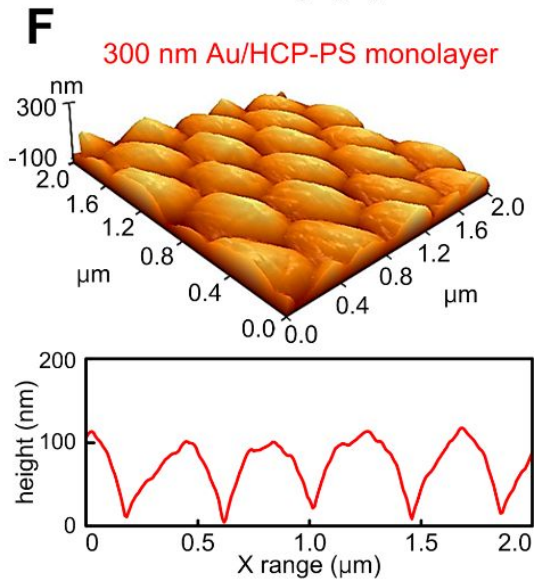

Figure S2. AFM tapping-mode topographical images of the Au/HCP-PS microsphere arrays on the $\mathrm{Si}$ substrate with Au thicknesses of (A) $50 \mathrm{~nm}$, (B) $100 \mathrm{~nm}$, (C) $150 \mathrm{~nm}$, (D) $200 \mathrm{~nm}$, (E) $250 \mathrm{~nm}$, and (F) $300 \mathrm{~nm}$.

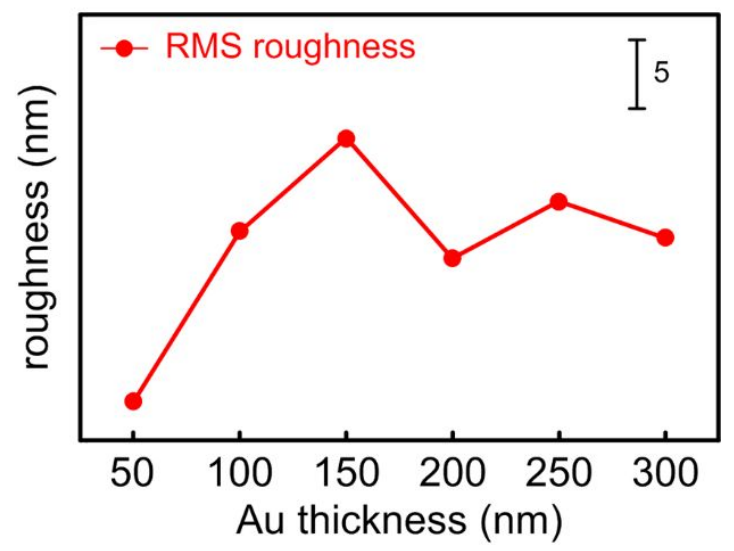

Figure S3. Plot of AFM-estimated RMS roughness with the Au/HCP-PS monolayer on the Si substrate with Au thicknesses of $50-300 \mathrm{~nm}$. 


\section{Wavelength characteristics of Au/HCP-PS monolayer}

Table S1. Optical depths $\left(d_{\text {opt }}\right)$ of the Au/HCP-PS monolayer with Au thicknesses of $50-300 \mathrm{~nm}$ at three representative Raman excitation wavelengths*

\begin{tabular}{llllll}
\hline \multicolumn{2}{l}{ Au thickness $(\mathbf{n m})$} & & \multicolumn{3}{c}{ Raman excitation wavelength } \\
\cline { 1 - 2 } \cline { 5 - 6 } Set & Estimation & & $\mathbf{5 3 2} \mathbf{~ n m}$ & $\mathbf{6 3 3} \mathbf{~ n m}$ & $\mathbf{7 8 5} \mathbf{~ n m}$ \\
\hline 50 & 67 & & 36.18 & 12.06 & 10.05 \\
100 & 126 & & 68.04 & 22.68 & 18.90 \\
150 & 172 & & 92.88 & 30.96 & 25.80 \\
200 & 223 & & 120.42 & 40.14 & 33.45 \\
250 & 239 & & 129.06 & 43.02 & 35.85 \\
300 & 304 & & 164.16 & 54.72 & 45.60 \\
\hline
\end{tabular}

${ }^{*}$ The refractive indices of $\mathrm{Au}$ at 532,633 , and $785 \mathrm{~nm}$ were $0.54,0.18$, and 0.15 , respectively.
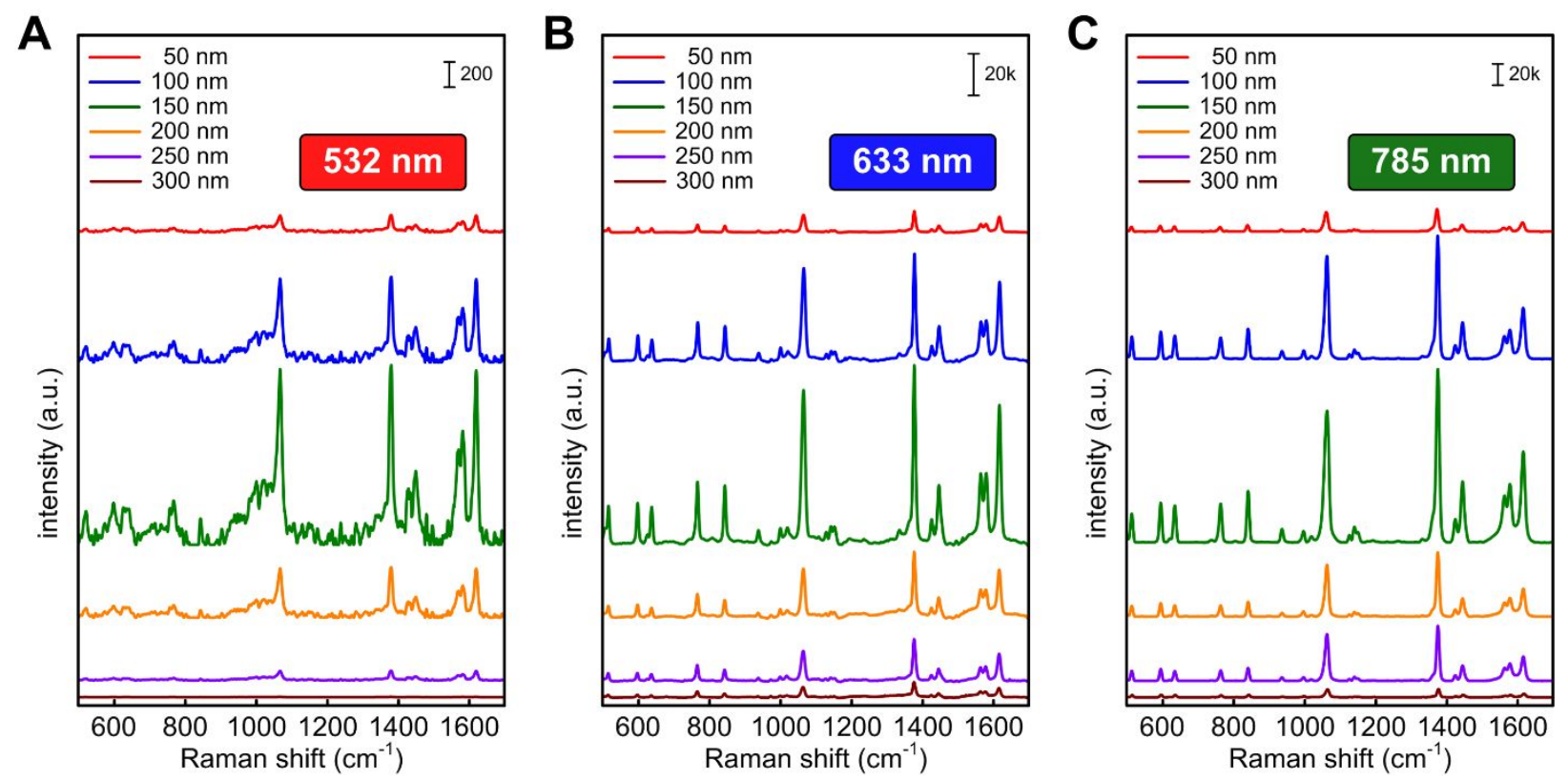

Figure S4. Variations in Raman intensities with Au thicknesses of $50-300 \mathrm{~nm}$ at excitation wavelengths of (A) 532, (B) 633, and (C) $785 \mathrm{~nm}$. 


\section{D FDTD models of Au/HCP-PS monolayer}
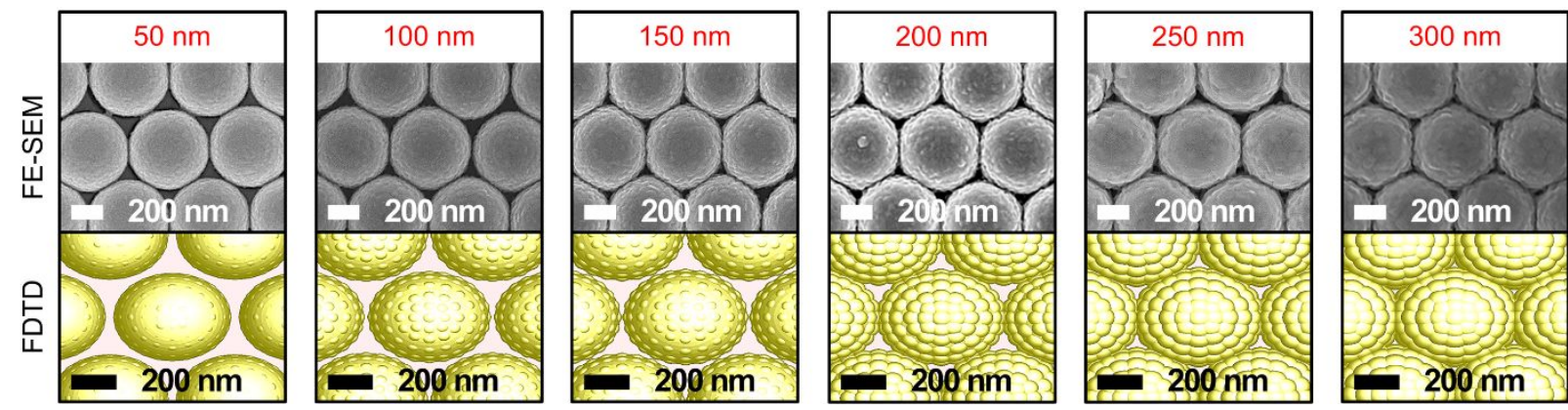

Figure S5. 3D FDTD models of the Au/HCP-PS microspheres approximated from FE-SEM images with Au thicknesses of $50-300 \mathrm{~nm}$.

\section{SERS-EF calculation of Au/HCP-PS monolayer}

Table S2. SERS performance of as-optimized 150-nm Au/HCP-PS monolayer at three representative Raman excitation wavelengths

\begin{tabular}{llll}
\hline SERS performance & \multicolumn{3}{l}{ Raman excitation wavelength } \\
\cline { 2 - 4 } & $\mathbf{5 3 2} \mathbf{~} \mathbf{m}$ & $\mathbf{6 3 3} \mathbf{~ n m}$ & $\mathbf{7 8 5} \mathbf{~} \mathbf{~}$ \\
\hline Raman intensity at 150-nm Au thickness (Fig. 2A) & $1.4 \times 10^{3}$ & $8.6 \times 10^{4}$ & $1.6 \times 10^{5}$ \\
$\mid$ E| $\left.\right|^{4}$-approximation SERS EF at 150-nm Au thickness (Fig. 2B) & $6.2 \times 10^{3}$ & $4.3 \times 10^{6}$ & $4.8 \times 10^{7}$ \\
& $2.9 \times 10^{4, * *}$ & \\
LOD (Fig. 3B) & & $100 \mathrm{fM}$ \\
SERS EF at analytical chemistry (Fig. 3C) & & $5.4 \times 10^{10}$ \\
Linear correlation for 1 mM to 100 fM, R ${ }^{2}$ (Fig. 3D) & & 0.9969 \\
Reproducibility, RSD & & $6.5 \%$ \\
Repeatability, RSD & & $3.5 \%$ \\
\hline
\end{tabular}

${ }^{*}$ Raman intensities at $1378 \mathrm{~cm}^{-1}$ of $1 \mathrm{mM}$ 2-NAT.

**The highest EM field distribution was observed at an Au thickness of 200-nm. 

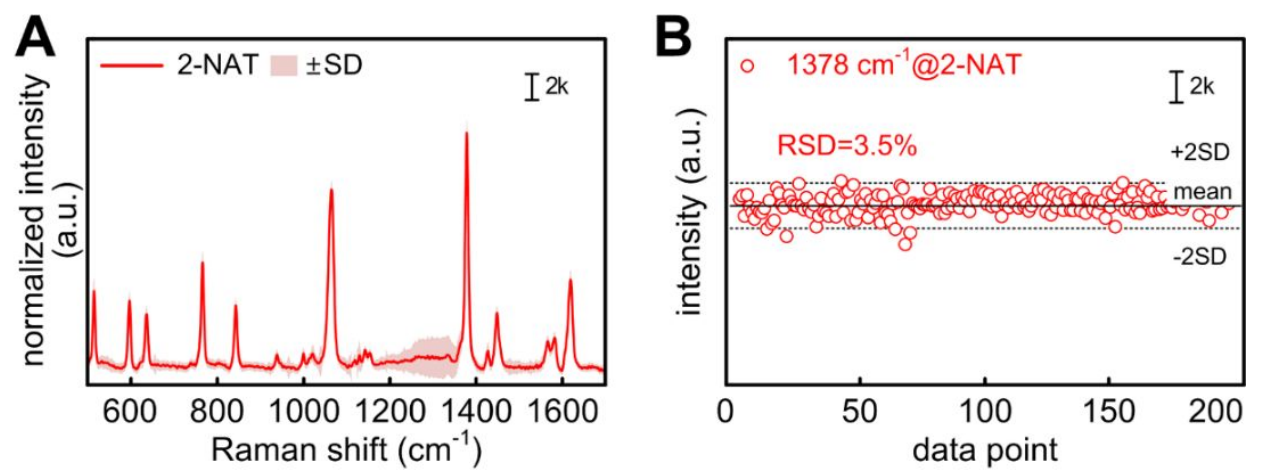

Figure S6. (A) Variations in Raman intensities for 200 Raman spectra of 1- $\mu \mathrm{M} 2-\mathrm{NAT}$ at the same measurement point. (B) High repeatability with $\mathrm{RSD}=3.5 \%$ at $1,378 \mathrm{~cm}^{-1}$ peak.

\section{Plasmonic Au/HCP-PS monolayer biosensor chip}
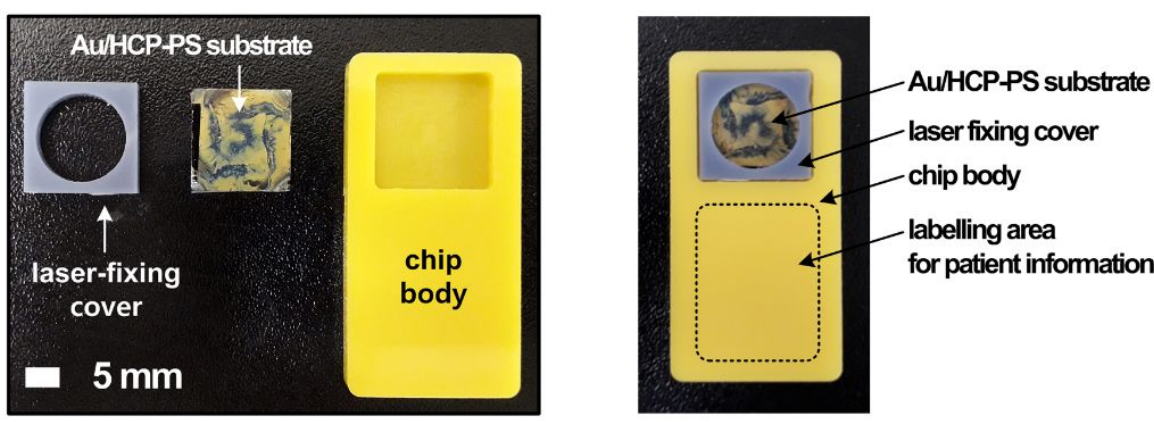

Figure S7. Photo of the Au/HCP-PS monolayer SERS biosensor chip. 


\section{PC loading profiles of PCA model for discrimination between healthy control and}

\section{breast cancer}
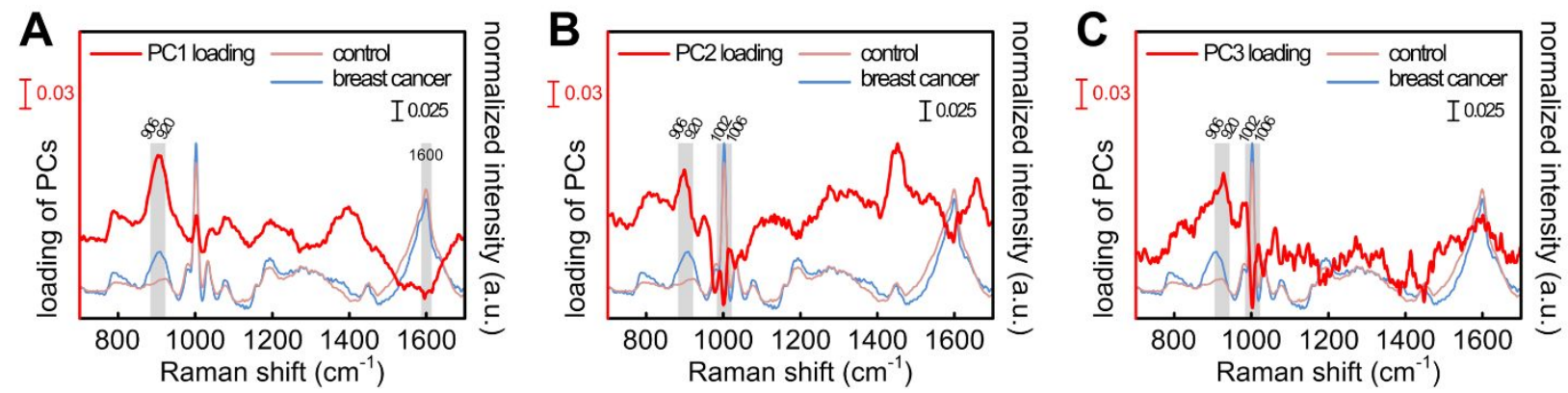

Figure S8. Loading profiles of (A) PC1, (B) PC2, and (C) PC3, calculated from 100 Raman spectra of 50 healthy controls and 50 patients with breast cancer.

\section{Practical application of plasmonic Au/HCP-PS monolayer biosensor chip}
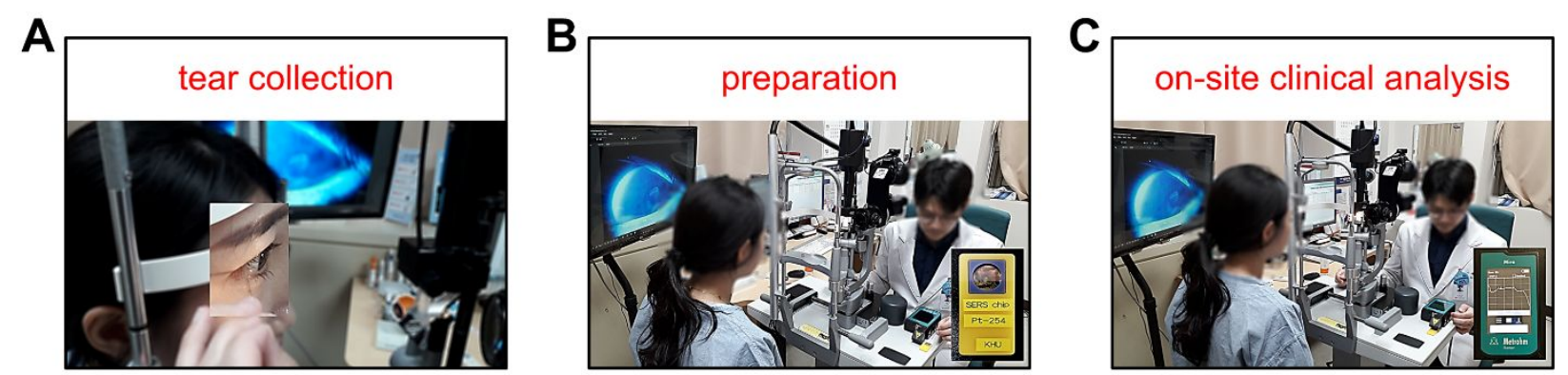

Figure S9. Asymptomatic breast cancer diagnosis at clinical sites using human tears. (A) Tear collection, (B) drop coating deposition, and (C) on-site clinical analysis. 